\section{Anderson's private screening}

\section{By Kai-Jye Lou, Staff Writer}

Less than two years after The University of Texas MD Anderson Cancer Center carved up the IP for its in vivo phage display screening platform, the two biotech licensees have preclinical proof-of-concept data and preliminary patient validation for products based on the technology. Alvos Therapeutics Inc. is now determining optimal peptidepayload combinations for use in cancer, while Ablaris Therapeutics Inc. is developing follow-on formulations of its lead compound for obesity-related indications.

MD Anderson spent more than a decade developing its in vivo patient-based screening platform. ${ }^{1}$ The technology involves injecting terminally ill cancer patients with a large-scale phage display library of peptides that circulate for a short time before life support is terminated. Follow-up biopsies and phage rescue reveal which peptides home to which tissues and the receptors the peptides bind.

Peptides significantly enriched in diseased tissues could be used to deliver a therapeutic payload to those tissues. According to Alvos COO Pete Leone, screening in patients is important because, unlike conventional screening platforms, it can determine whether a target is on the surface or inside of a human cell. This is difficult to determine with proteomic approaches and cadaveric tissue samples, he said.

Late last month, the MD Anderson group published a study in the Proceedings of the National Academy of Sciences that provided additional human validation for the screening platform licensed to Alvos and Ablaris. ${ }^{2}$ The study identified two ligand-receptor pairs common to the vasculature of multiple human tissues and a pair specific to bone metastases.

The study also provided mechanistic and functional validation for the target of Ablaris' lead compound adipotide, a peptidomimetic consisting of a prohibitin 1 (PHB; PHB1)-targeting peptide coupled to a proapoptotic helical peptide that disrupts mitochondrial membranes. The researchers found an annexin A2 (ANXA2)-PHB ligand-receptor pair specific to the vasculature for normal white adipose tissues and showed that the targeting peptide domain of PHB mimicked a region on ANXA2.

This month, the academics published in Science Translational Medicine that adipotide caused weight loss and increased insulin resistance in obese nonhuman primates. ${ }^{3}$

The two studies were co-led by Renata Pasqualini and Wadih Arap, who are both professors in the Department of Genitourinary Medical Oncology at MD Anderson and cofounders of Alvos and Ablaris.

\section{Ablaris in obesity}

In the Science Translational Medicine study, the MD Anderson researchers showed that obese nonhuman primates receiving daily adipotide injections for 28 days had lower body weight, BMI, abdominal circumference and total body fat percentage than controls given saline $(p<0.0001$ for all). Adipotide also improved insulin resistance compared with saline $(p=0.006)$.

"The biggest impact of the Science Translational Medicine study is in demonstrating the pharmacological efficacy of our lead compound in a primate system," said James Hulvat, director of R\&D at Ablaris and coauthor on the paper. "Most drug candidates in our space fail when going from rodents to humans or other primates because the physiology of food intake and metabolism are very different."

Because white adipose tissue produces factors that can promote prostate cancer growth, ${ }^{4,5} \mathrm{MD}$ Anderson has submitted an IND for a Phase I trial of adipotide in obese men with castration-resistant prostate cancer. The center plans to start patient recruitment early next year.

In this trial, Hulvat expects adipotide will be dosed daily for 28 days via subcutaneous injection.

Ablaris is taking a different tack with the molecule and is developing adipotide as a lead-in therapy to treat morbidly obese patients planning to undergo bariatric surgery. The compound is in preclinical development for the indication.

According to Hulvat, a decrease in body weight prior to bariatric surgery is associated with a decrease in surgery-related complications and mortality. ${ }^{6}$

The dosing schedule for the MD Anderson-sponsored-trial would not be optimal for broader obesity-related indications, and as a result Ablaris also is developing follow-on formulations of adipotide.

The goal, said Hulvat, will be formulations that could be dosed weekly or given as a subdermal implant. The company also is working to broaden adipotide's therapeutic index by improving the adipotide-receptor interaction to increase potency and by altering the compound's renal clearance to decrease toxicity risk.

The company hopes to submit an IND for a follow-on adipotide formulation in late 2012 or early 2013.

Parent company Arrowhead Research Corp. launched Ablaris in December 2010 to commercialize the technology licensed from MD Anderson. Arrowhead holds a majority stake in Ablaris.

\section{Alvos in oncology}

While Ablaris is focused on obesity, Alvos is using peptides identified with the MD Anderson screening platform to target therapeutic 


\section{ANALYSIS}

\section{TRANSLATIONAL NOTES}

payloads to cancer cells. The company also is using the platform to identify additional targeting peptides and their corresponding receptors.

\section{"The biggest impact of} the Science Translational Medicine study is in demonstrating the pharmacological efficacy of our lead compound in a primate system."

- James Hulvat,

Ablaris Therapeutics Inc.

Alvos was founded in July 2010 as Mercator Therapeutics. The following month, the biotech closed a $\$ 2$ million venture round and announced the licensing deal with MD Anderson.

Alvos' programs include peptides that target IL-11 receptor, heat shock $70 \mathrm{kDa}$ protein 5 (glucose-regulated protein, $78 \mathrm{kDa}$; HspA5; GRP78) and v-crk sarcoma virus CT10 oncogene homolog (avian)-like (CRKL). Leone said Alvos is determining the optimal peptide-payload combinations for various tumor types and is about two years away from starting a clinical trial.

The company's plan is to run side-by-side comparisons to show that linking approved and new cancer drugs to Alvos' targeting peptides will improve safety and efficacy.

Alvos' rights to the MD Anderson technology include all indications outside weight loss and obesity-related conditions. Leone said the biotech is in early discussions with two companies for candidate homing peptides that could be useful in cardiovascular diseases and in a muscle wasting disorder.

"We'll provide the molecule that gets the payload to the target cells, and our partners in these other spaces will provide the payload they wish to deliver," he told SciBX. "We've also identified several candidate peptides that could be useful for delivering a therapeutic payload across the blood brain barrier and another that could potentially deliver compounds to pancreatic cells."

According to Leone, the MD Anderson group has discovered peptides that home specifically to about 30 different tissues and their corresponding receptors.

Lou, K.-J. SciBX 4(45); doi:10.1038/scibx.2011.1257

Published online Nov. 17, 2011

\section{REFERENCES}

1. Arap, W. et al. Nat. Med. 8, 121-127 (2002)

2. Staquicini, F.I. et al. Proc. Natl. Acad. Sci. USA; published online Oct. 24, 2011; doi:10.1073/pnas.1114503108

Contact: Wadih Arap, The University of Texas MD Anderson Cancer Center, Houston, Texas

e-mail: warap@mdanderson.org

Contact: Renata Pasqualini, same affiliation as above e-mail: rpasqual@mdanderson.org

Contact: Richard L. Sidman, Beth Israel Deaconess Medical Center and Harvard Medical School, Boston, Mass.

e-mail: richard_sidman@hms.harvard.edu

3. Barnhart, K.F. et al. Sci. Transl. Med.; published online Nov. 9, 2011; doi:10.1126/scitransImed.3002621

Contact: Renata Pasqualini, University of Texas MD Anderson Cancer Center, Houston, Texas e-mail: rpasqual@mdanderson.org

Contact: Wadih Arap, same affiliation as above e-mail: warap@mdanderson.org

4. Hsing, A.W. et al. Am. J. Clin. Nutr. 86, s843-s857 (2007)

5. Mistry, T. et al. Eur. Urol. 52, 46-53 (2007)

6. Benotti, P.N. et al. Arch. Surg. 144, 1150-1155 (2009)

\section{COMPANIES AND INSTITUTIONS MENTIONED}

Ablaris Therapeutics Inc., Pasadena, Calif.

Alvos Therapeutics Inc., Wellesley, Mass.

Arrowhead Research Corp. (NASDAQ:ARWR), Pasadena, Calif. The University of Texas MD Anderson Cancer Center, Houston, Texas 